\title{
Motion Planning for a Humanoid Mobile Manipulator System
}

\author{
Yan Wei, Wei Jiang, Ahmed Rahmani \\ CRIStAL, UMR CNRS 9189, Ecole Centrale de Lille \\ Villeneuve d'Ascq, 59650, France \\ wytt0324@gmail.com,wjiang.lab@gmail.com \\ Qiang Zhan \\ Robot Research Institute, Beihang University \\ Beijing, 100191, China
}

\begin{abstract}
A high redundant non-holonomic humanoid mobile dual-arm manipulator system (MDAMS) is presented in this paper where the motion planning to realize "humanlike" autonomous navigation and manipulation tasks is studied. Firstly, an improved MaxiMin NSGA-II algorithm, which optimizes five objective functions to solve the problems of singularity, redundancy, and coupling between mobile base and manipulator simultaneously, is proposed to design the optimal pose to manipulate the target object. Then, in order to link the initial pose and that optimal pose, an off-line motion planning algorithm is designed. In detail, an efficient direct-connect bidirectional RRT and gradient descent algorithm is proposed to reduce the sampled nodes largely, and a geometric optimization method is proposed for path pruning. Besides, head forward behaviors are realized by calculating the reasonable orientations and assigning them to the mobile base to improve the quality of human-robot interaction. Thirdly, the extension to online planning is done by introducing real-time sensing, collision-test and control cycles to update robotic motion in dynamic environments. Fourthly, an EEs' via-point-based multi-objective genetic algorithm (MOGA) is proposed to design the "human-like" viaposes by optimizing four objective functions. Finally, numerous simulations are presented to validate the effectiveness of proposed algorithms.
\end{abstract}

Keywords: Humanoid mobile manipulator; On-line motion planning; MaxiMin NSGA-II; Multi-objective optimization; Path optimization.

\section{Introduction}

Industrial manipulators have been widely used to do some repetitive tasks, but they are usually fixed manipulators in structured environments with predefined task policies and control inputs. Recently, more and more mobile manipulators are utilized in fields from personal assistance to military applications ${ }^{1-5}$. However, personal robots, normally humanoid mobile manipulators, are required to work automatically in unstructured environments with humans. In order to improve the quality of human-robot interaction, robots' behaviors should be predictable and natural. The existing personal assistant, entertainment, accompany or guidance

* Corresponding author. 
robots (e.g., sweeping robots, $A M I^{1}$ and $P$ epper ${ }^{2}$ ) are usually mono-functional, nonprogrammable, non-interactive or too specialized for non-expert users. Therefore, the research of multi-functional, completely autonomous and sustainable personal robots is still open and challenging. ${ }^{5,6}$

Many motion planning methods ${ }^{7-10}$ for multiple degrees of freedom (DoFs) robots have been presented, but they are incapable of solving motion planning problems under multiple constraints. Direct and inverse kinematics (IK)-based indirect motion planning methods for multi-DoFs manipulators ${ }^{11-19}$ have been proposed. However, for direct methods, the EE's motion is difficult to predict. For IK-based indirect methods, though EE's motion is guaranteed, they need to optimize a predefined objective function which must be continuous ${ }^{17-19}$, and require the inverse Jacobian calculation which leads to singularity problem and results in unpredictable behaviors due to the high non-linearity between the task and joint spaces. In order to overcome these shortcomings, evolutionary algorithms represented by genetic algorithms (GAs) are used ${ }^{20-25}$ since GA has no constraints on cost functions (they can be discontinuous, not differentiable, stochastic, or highly nonlinear), no need of calculating inverse Jacobian, and is capable of optimizing (maximizing and minimizing simultaneously) multiple objectives, etc. ${ }^{26}$ In reality, the multiple objectives usually conflict with each other. By defining a combined fitness function, MOGAs can also take various objectives into account at the same time. For instance, the multi-objectives are EE's positioning error and joint displacement for a redundant manipulator ${ }^{22}$ and for a mobile manipulator ${ }^{24}$, mobile base and joint's displacements for a mobile manipulator ${ }^{23}$, the navigation length, path-obstacles intersection and accumulated change of mobile base's orientation for a mobile vehicle ${ }^{25}$, etc. However, most of them do not study the coordination between the position-orientation of mobile base and manipulator's configuration, and not mention the "human-like" motion design.

On the other hand, naturally, the presence of multiple objectives can result in a set of optimal solutions (known as Pareto-optimal solutions). The problem is that the combined fitness function-based MOGAs can only generate one optimal solution and needs to specify the weighting coefficients in advance, which means that this algorithm loses the diversity. In order to preserve the diversity, Srinivas ${ }^{27}$ proposed a non-dominated sorting genetic algorithm (NSGA) searching for the optimal solution in multiple directions. As a result, multiple solutions are generalized to form a Pareto-optimal set. However, NSGA needs to specify a predefined sharing parameter. To release this constraint, $\mathrm{Deb}^{28}$ proposed the NSGA-II by introducing a fast-non-dominated-sort scheme and a crowding distance assignment. In the above two works, Pareto-optimal solutions in each generation are not well distributed. Hence, Pires ${ }^{29}$ introduced the MaxiMin sorting scheme into NSGA-II where only the last selected front is sorted, but the previous fronts are not. This is why we propose an improved MaxiMin NSGA-II algorithm in which not only the last selected front but all the selected fronts are sorted using the MaxiMin sorting scheme. In 
this way, the probability of inheriting good genes is improved as much as possible at each generation.

Another problem is that since the off-line designed motion may fail by colliding with obstacles in dynamic environments, on-line motion planning methods, especially RRTs-based methods, attract great interest due to their high efficiency. ${ }^{30-32}$ As many motion planning methods generate low quality paths, path pruning techniques are proposed based on the medial axis. ${ }^{33,34}$ However, they require calculating the medial axis in the collision-free configuration space $C_{\text {free }}$ which is complex. The $\mathrm{RRT}^{* 31}$ consists of reconnect and regrow processes and only guarantees local optimal path. The Informed-RRT ${ }^{* 32}$ is asymptotically optimal but it needs to evaluate the whole path which is time-consuming and unnecessary. Besides, they mainly focus on motion planning for mobile or free-flying robots and do not consider the non-holonomic constraints. ${ }^{31,32}$ Even thought there exist studies on non-holonomic mobile vehicles ${ }^{35}$, they do not take into account the heading angle which is very important for non-holonomic humanoid mobile manipulators studied in this paper.

Theoretically speaking, the above mentioned works can be applied to high DoFs robots, but their effectiveness will decrease and they are incompetent to solve multiple constrained problems. ${ }^{30-35}$ Many researchers work on motion planning for multi-DoFs manipulators ${ }^{36,42}$ in real time. Lee $^{36}$ proposed a virtual roadmap-based on-line trajectory generation method for a dual-arm robot. Berg ${ }^{37}$ planned the motion in state-time space using roadmap and A*. Singularity avoidance of a mobile surgery assistant was studied ${ }^{37}$ via a penalty function, but the EE's path is needed in advance. Yang ${ }^{39}$ introduced a configuration re-planning method for a fixed manipulator in dynamic environments. However, it requires calculating inverse Jacobian and the desired configuration must be known. Chen ${ }^{40}$ designed a joint velocity correction term to the manipulator's joint trajectory. Xin ${ }^{41}$ introduced an escape velocity and projected it onto the null space for redundant arms. $\operatorname{Han}^{42}$ used the distance calculation and discrete detection for robot arms. However, they do not deal with the multiple constrains or "human-like" behaviors. In order to realize "human-like" behaviors, a heuristic method was presented for a multi-DoFs humanoid robot arm using the RRT* algorithm directly in task space. ${ }^{43}$ However, only the EE and elbow are considered as two hierarchical control points. In fact, as the number of control points increases, the hierarchical motion planning method will become much more complicated. Vannoy ${ }^{44}$ generated a population of trajectories based on the fitness evaluation for mobile manipulators in dynamic environments with unknown moving obstacles; however, this method is time-consuming. Zhao ${ }^{45}$ proposed a motion-decision algorithm to realize "human-like" movements only for a redundant arm. Besides, many reasoning methods, like analytic and fuzzy logic methods, have been introduced to achieve "human-like" behaviors. ${ }^{46-49}$ However, the specific consideration and rules for different scenarios are required which are complicated for high DoFs systems.

To summarize, in the above-mentioned methods, the desired pose or the EE's path needs to be known in advance. However, they can be unknown in this paper. 
The "human-like" behavior design for the humanoid robot is still an open problem. In this paper, only the EEs' desired positions-orientations are known. The optimal pose (see Definition 1) and the motions in task and joint space are to be designed. The objective is to make the MDAMS be "human-like" and "natural" as much as possible in both their appearance and behaviors to guarantee the quality of humanrobot interaction. The main contributions are listed as follows:

- An improved MaxiMin NSGA-II Algorithm 1 is proposed in Section 3, It is used to design the optimal pose given only EEs' desired positions-orientations. Five objective functions are optimized simultaneously to achieve "natural" behaviors. The position-orientation of mobile base and the configuration of manipulator are planned at the same time.

- A fast off-line motion planning Algorithm 2 is given in Section 4 considering the optimal pose has been designed by Algorithm 1

- A direct-connect bidirectional RRT and gradient descent sampling process is proposed to improve the performance of RRTs in Section 4.1

- An efficient geometric optimization method pruning the sampled path via node rejection and node adjustment, is proposed to always guarantee the shortest consistent path for repeated tasks in Section 4.2 .

- An on-line motion planning Algorithm 3 is designed in Section 5 by introducing on-line sensing, collision-test and control cycles with the dynamic obstacles being treated in real time. In order to take the via-poses into consideration, an EEs' viapoint-based MOGA algorithm is presented. Four objective functions are defined to optimize the candidate via-poses corresponding to the EEs' via-points.

Besides, the forward kinematics for MDAMS is established using the modified Denavit-Hartenberg (MDH) method in Section 2, and a number of motion planning simulations are presented in Section 6 .

\section{System Modeling of MDAMS}

The structure of the designed MDAMS is illustrated in Fig. 1 (a). It has two armhand subsystems sharing one common waist and one common non-holonomic mobile base. The MDH method is used to characterize the motion of frame $\Sigma_{i}$ in $\Sigma_{i-1}$ via the transformation matrix:

$$
T_{i-1}^{i}=\operatorname{Rot}\left(x, \alpha_{i-1}\right) \operatorname{Trans}\left(x, a_{i-1}\right) \operatorname{Rot}\left(z, \theta_{i}\right) \operatorname{Trans}\left(z, d_{i}\right)=\left[\begin{array}{cc}
R_{i-1}^{i} & P_{i-1}^{i} \\
0 & 1
\end{array}\right]
$$

through a sequence of rotations $\operatorname{Rot}()$ and translations $\operatorname{Trans}()$ with only four parameters $\alpha_{i-1}, a_{i-1}, \theta_{i}$ and $d_{i} . R_{i-1}^{i}$ and $P_{i-1}^{i}$ are the rotation matrix and position vector of $\Sigma_{i}$ in $\Sigma_{i-1}$, respectively.

Apply the MDH method to the designed MDAMS, 22 frames are defined without considering the neck, head and fingers ${ }^{50}$. Specifically, $\Sigma_{0}$ is the world frame; $\Sigma_{i}$ $(i=1, \ldots, 19)$ is the frame with its origin locating at point $P_{i} ; P_{e}$ and $P_{e^{\prime}}$ are the 
(a)

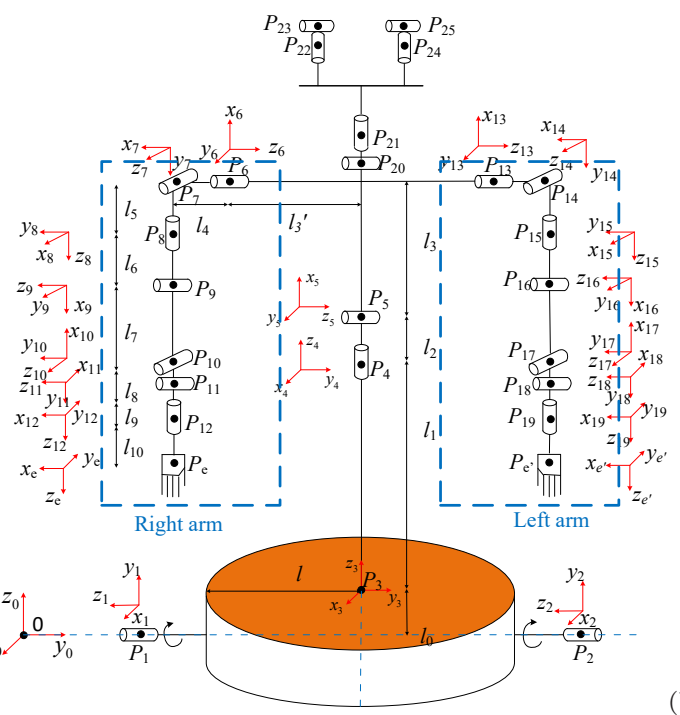

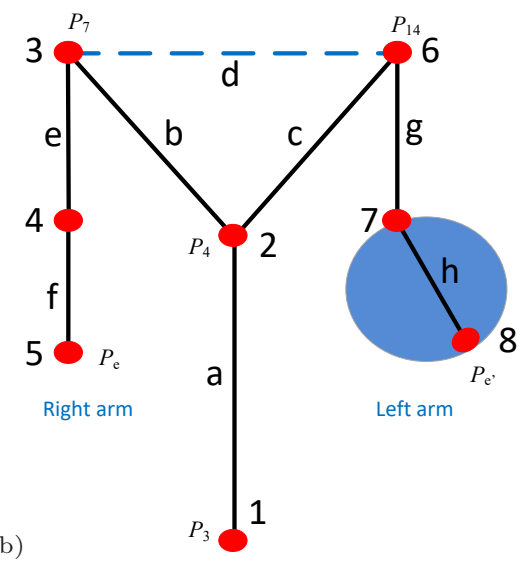

Fig. 1. Mechanical description of MDAMS. (a) System structure; (b) System simplification.

palm centers (EEs); $\Sigma_{e}$ and $\Sigma_{e^{\prime}}$ are EEs' frames with a translation along axis $z_{12}$ and $z_{19}$, respectively. In particular, frame $\Sigma_{3}$ is attached to the mobile base which is described by $\boldsymbol{\theta}_{m}=(x, y, \phi)$ where $(x, y)$ and $\phi$ are mobile base's position and orientation in $\Sigma_{0}$, respectively. $l_{0}$ is the translation of $\Sigma_{3}$ along axis $z_{0}$ of $\Sigma_{0}$ and $l_{k}(k=1, \ldots, 10)$ characterizes the geometric dimension (see Table 1) of MDAMS in which the variables are defined as follows:

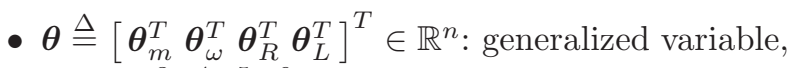

- $T_{R}=T_{0}^{3} T_{3}^{4} T_{4}^{5} T_{5}^{6} T_{6}^{e}\left(\boldsymbol{\theta}_{m}, \boldsymbol{\theta}_{w}, \boldsymbol{\theta}_{R}\right)$ : transformation matrix of the right EE in $\Sigma_{0}$,

- $T_{L}=T_{0}^{3} T_{3}^{4} T_{4}^{5} T_{5}^{13} T_{13}^{e^{\prime}}\left(\boldsymbol{\theta}_{m}, \boldsymbol{\theta}_{w}, \boldsymbol{\theta}_{L}\right)$ : transformation matrix of the left EE in $\Sigma_{0}$,

- $T_{R b}=T_{3}^{4} T_{4}^{5} T_{5}^{6} T_{6}^{e}\left(\boldsymbol{\theta}_{w}, \boldsymbol{\theta}_{R}\right)$ : transformation matrix of the right EE in $\Sigma_{3}$,

- $T_{L b}=T_{3}^{4} T_{4}^{5} T_{5}^{13} T_{13}^{e^{\prime}}\left(\boldsymbol{\theta}_{w}, \boldsymbol{\theta}_{L}\right)$ : transformation matrix of the left EE in $\Sigma_{3}$,

- $\dot{x}_{R}=\left[J_{v_{R}} \mathbf{0}_{n_{L}}\right] \dot{\boldsymbol{\theta}}=J_{R} \dot{\boldsymbol{\theta}} \in \mathbb{R}^{m}$ : linear velocity of the right EE in $\Sigma_{0}$,

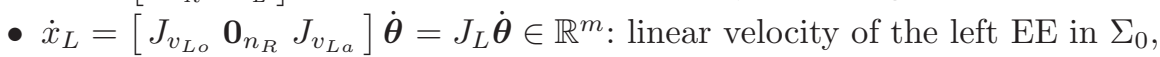

- $\omega=\left[\omega_{R}^{T} \omega_{L}^{T}\right]^{T}=\left[J_{\omega_{R}}^{T} J_{\omega_{L}}^{T}\right]^{T} \dot{\boldsymbol{\theta}}=J_{\omega} \dot{\boldsymbol{\theta}} \in \mathbb{R}^{2 m}$ : EEs' angular velocities in $\Sigma_{0}$,

where $\boldsymbol{\theta}_{w} \in \mathbb{R}^{n_{w}}, \boldsymbol{\theta}_{R} \in \mathbb{R}^{n_{R}}$ and $\boldsymbol{\theta}_{L} \in \mathbb{R}^{n_{L}}$ represent the joints in waist, right and left arms, respectively. $\left(\boldsymbol{\theta}_{w}, \boldsymbol{\theta}_{R}, \boldsymbol{\theta}_{L}\right)$ represents the configuration of manipulator. $J_{v_{R}} \in \mathbb{R}^{m \times\left(n_{m}+n_{w}+n_{R}\right)}$ is the right EE's Jacobian in $\Sigma_{0} ; J_{v_{L o}} \in \mathbb{R}^{m \times\left(n_{m}+n_{w}\right)}$ and $J_{v_{L a}} \in \mathbb{R}^{m \times n_{R}}$ are the left EE's Jacobians in $\Sigma_{0} ; J_{\omega_{i}}(i=R, L)$ is the EE's orientation Jacobian. In this paper, $n_{m}=3, n_{w}=2, n_{R}=n_{L}=7, n=n_{m}+n_{w}+$ $n_{R}+n_{L}=19$ and $m=3$.

Definition 1. (Pose and Optimal pose) Throughout this paper, we note pose 
Table 1. Robot's dimension.

\begin{tabular}{l|l|l|l|l|l|l|l|l|l|l|l}
\hline $\mathrm{l}$ & $l_{0}$ & $l_{1}$ & $l_{2}$ & $l_{3} / l_{3}^{\prime}$ & $l_{4}$ & $l_{5}$ & $l_{6}$ & $l_{7}$ & $l_{8}$ & $l_{9}$ & $l_{10}$ \\
\hline 0.16 & 0.262 & 0.45 & 0.15 & 0.3 & 0.15 & 0.2 & 0.05 & 0.2 & 0.05 & 0.05 & 0.05 \\
\hline
\end{tabular}

as the position-orientation of mobile base and the configuration of upper manipulator (i.e. $\boldsymbol{\theta}$ ) for the designed MDAMS. $\boldsymbol{\theta}_{o p}$ denotes the optimal pose which reaches EEs' desired positions-orientations $\boldsymbol{X}_{E E}$ under multiple constraints.

Suppose that the initial pose and $\boldsymbol{X}_{E E}$ of MDAMS are known. In the rest of this paper, optimal pose design, off-line and on-line motion planning problems for MDAMS are investigated.

\section{Optimal Pose Design}

The GA is a popular method for solving optimization problems based on natural genetics and selection mechanics ${ }^{51}$. A GA drives a population which is composed of many individuals that are usually represented by a set of parameters (known as chromosomes), to evolve under specified selection rules to a state that maximizes the fitness function. The population of individuals (possible solutions) which is initialized randomly, is modified repeatedly through selection, crossover and mutation operators. At each step, the GA selects good individuals to be parents from the current population, and uses them to produce the offspring for the next generation (crossover). Random changes are introduced to the population by means of mutation operator. The population evolves towards an optimal solution over successive generations.

\subsection{Objective functions}

Due to the high redundancy, there exist numerous joints combinations given EEs' desired positions-orientations $\boldsymbol{X}_{E E}$, and there is always a preference. Instead of only taking into account EE's positioning accuracy or joint displacement, five objective functions are considered simultaneously as follows:

- EE's positioning accuracy: $f_{1}(\boldsymbol{\theta})=\left\|x_{R}(\boldsymbol{\theta})-x_{R d}\right\|+\left\|x_{L}(\boldsymbol{\theta})-x_{L d}\right\|$, where $x_{R d}, x_{L d}, x_{R}(\boldsymbol{\theta})$ and $x_{L}(\boldsymbol{\theta})$ are the desired and real positions of right and left EEs, respectively.

- EE's orientation tracking accuracy: $f_{2}(\boldsymbol{\theta})=\left\|e_{q_{R}}(\boldsymbol{\theta})\right\|+\left\|e_{q_{L}}(\boldsymbol{\theta})\right\|$, where $e_{q_{R}}(\boldsymbol{\theta})$ and $e_{q_{L}}(\boldsymbol{\theta})$ are respectively the orientation tracking errors of right and left EEs using the unit quaterion representation. ${ }^{52}$

- EE's manipulability: Apart from reaching $\boldsymbol{X}_{E E}$, the additional criteria is required to achieve the "human-like" behaviors. The manipulability ability describes the distance away from robot's singular configuration. The usually used criterion is $\Omega=\sqrt{\operatorname{det}\left(J(\boldsymbol{\theta}) J^{T}(\boldsymbol{\theta})\right)}$ or $\Omega=\frac{\sigma_{\max }(J(\boldsymbol{\theta}))}{\sigma_{\min }(J(\boldsymbol{\theta}))}$ where $J(\boldsymbol{\theta})$ is EE's Jacobian and $\sigma$ is the singular value of $J(\boldsymbol{\theta})$. Then, the following objective function 
is given:

$f_{3}(\boldsymbol{\theta})=-\left(\Omega_{R}(\boldsymbol{\theta})+\Omega_{L}(\boldsymbol{\theta})\right)$, where $\Omega_{R}(\boldsymbol{\theta})$ and $\Omega_{L}(\boldsymbol{\theta})$ are the manipulability measures of right and left EEs, respectively.

- Joint displacement: In order to save energy, the least joint displacement is required. A mass-based joint displacement objective function is proposed as follows: $f_{4}(\boldsymbol{\theta})=\frac{1}{n} \sum_{i=1}^{n} W_{i} \frac{\boldsymbol{\theta}_{i}-\boldsymbol{\theta}_{\text {imin }}}{\boldsymbol{\theta}_{\text {imax }}-\boldsymbol{\theta}_{\text {imin }}}$, where $n$ is the dimension of the generalized variable $\boldsymbol{\theta}, \boldsymbol{\theta}_{i}$ is the $i$-th generalized variable, and $\boldsymbol{\theta}_{\text {imax }}$ and $\boldsymbol{\theta}_{\text {imin }}$ are the corresponding boundaries. $W_{i}=M_{i} / \sum_{j=1}^{n} M_{j}$ is mass-based weight, where $M_{i}=\sum_{k=i}^{n_{e}} m_{k}$, $m_{k}$ is the mass of link $k$, and $n_{e}$ is the index of EE (left or right). $f_{4}(\boldsymbol{\theta})$ means that the most heavy part has the largest difficulty to move.

- EE's displacement w.r.t. the mobile base: Since the above four functions mainly focus on the manipulator, the coordination between mobile base and manipulator is not studied. With regard to this, EEs' displacements in the frame $\Sigma_{3}$ of mobile base is $f_{5}(\boldsymbol{\theta})=\left\|x_{R b}(\boldsymbol{\theta})-x_{R b i}\right\|+\left\|x_{L b}(\boldsymbol{\theta})-x_{L b i}\right\|$, where $x_{R b i}$ and $x_{R b}(\boldsymbol{\theta})\left(x_{L b i}\right.$ and $\left.x_{L b}(\boldsymbol{\theta})\right)$ are the initial and desired positions of right (left) EE in $\Sigma_{3}$, respectively.

\subsection{Improved MaxiMin NSGA-II algorithm}

In this section, the improved MaxiMin NSGA-II Algorithm 1 is proposed to design the optimal pose $\boldsymbol{\theta}_{o p}$ by optimizing the above five objective functions. The chromosome is chosen as $\boldsymbol{\theta}=\left(x, y, \phi, \boldsymbol{\theta}_{\omega}, \boldsymbol{\theta}_{R}, \boldsymbol{\theta}_{L}\right)$ and its searching interval locates in $C_{\text {free }}$.

The whole process is illustrated in Fig. 2 and Algorithm 1, At the beginning, a parent population $P_{0}$ of size $N_{\text {pop }}$ is randomly created (line 1). Sort all the individuals $\left\{G_{i}, i=1, \ldots, N_{p o p}\right\}$ in $P_{0}$ according to their fitness values (line 2 , see Eq. (2)). The technique BasicGA, which employs the usual binary tournament selection, recombination and mutation operators, is used to create the first offspring population $Q_{0}$ of size $N_{\text {pop }}$ (line 3). The generation numeration is initialized $\iota=0$.

Then, a while loop is activated to evolve the Pareto-optimal solutions (lines 4 through 30). At the beginning of each generation $\iota$, a combined population $R_{\iota}=$

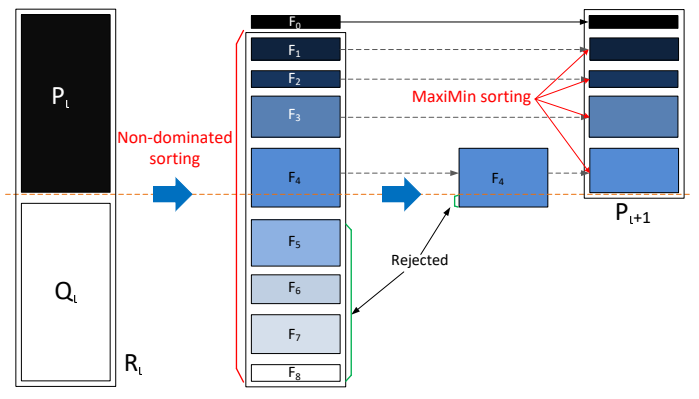

Fig. 2. Flowchart of the improved MaxiMin NSGA-II algorithm. 


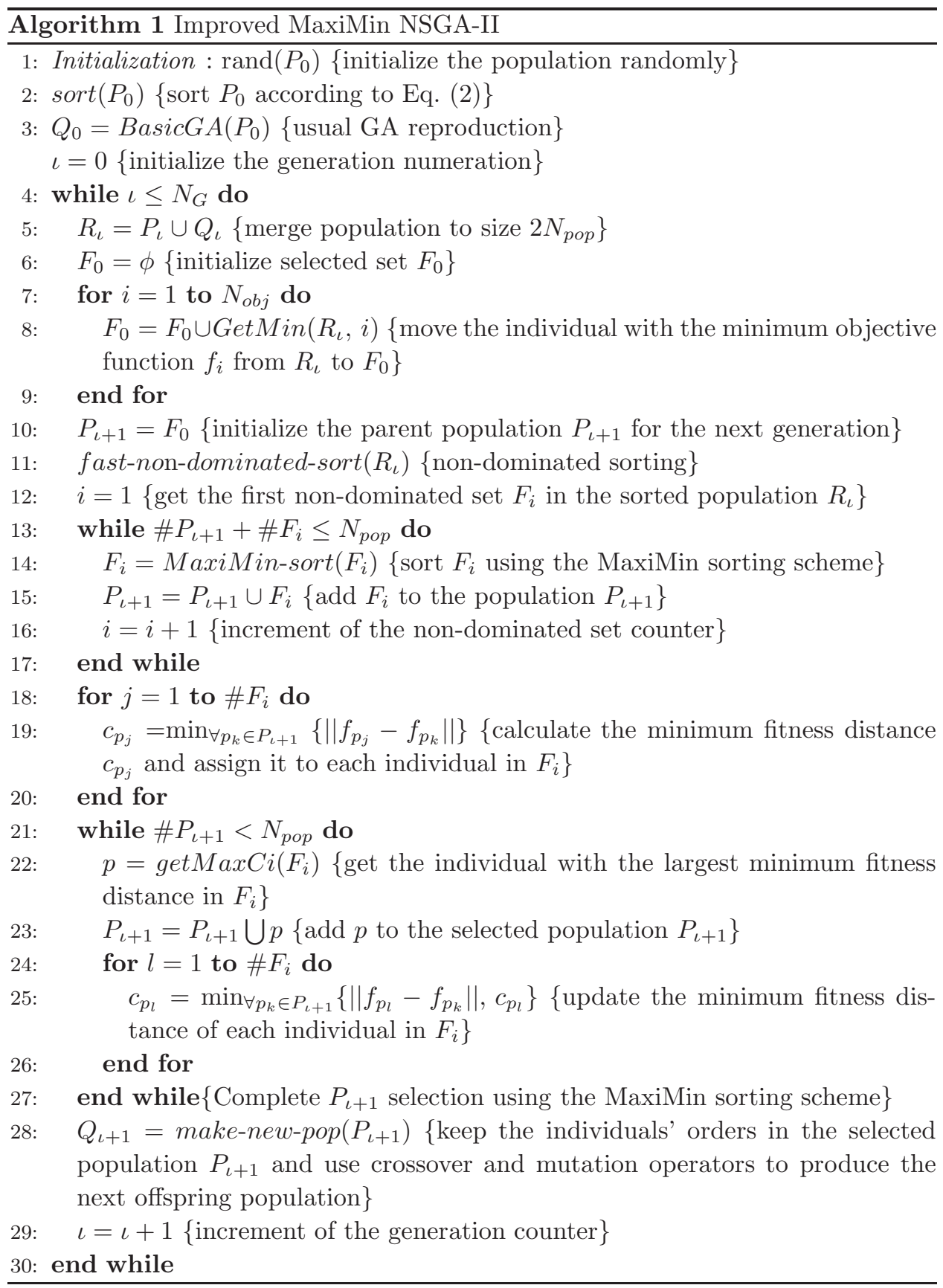

$P_{\iota} \cup Q_{\iota}$ of size $2 N_{\text {pop }}$ is formed (line 5). In $R_{\iota}$, get the individuals which have the minimum value for each objective function (lines 6 through 9 ) and initialize the next parent population $P_{\iota+1}$ (line 10). The rest individuals in $R_{\iota}$ are sorted using 
the fast non-dominated sorting scheme according to their non-domination ${ }^{24,28}$.

Afterwards, select the best non-dominated individuals set by set, and sort each set using the MaxiMin sorting scheme. In particular, if the number of individuals in the selected population $P_{\iota+1}$ and in the following set $F_{i}$ is smaller than $N_{\text {pop }}$ $\left(\# P_{\iota+1}+\# F_{i} \leq N_{\text {pop }}\right)$, continue the selection (lines 13 through 17); if not $\left(\# P_{\iota+1}+\right.$ $\# F_{i}>N_{\text {pop }}$ ), select the individuals one by one using the MaxiMin sorting scheme until obtaining $N_{\text {pop }}$ selected individuals (loops for and while, lines 18 through 20 and lines 21 through 27). Finally, the next offspring population is produced while keeping the order of the selected population $P_{\iota+1}$ and go to the next generation $(\iota=\iota+1$, line 29$)$.

The MaxiMin sorting scheme is described here. ${ }^{29}$ Firstly, the distance between each non-dominated individual $p_{j} \in F_{i}\left(j=1, \ldots, \# F_{i}\right)$ and the individuals already selected $p_{k} \in P_{\iota+1}\left(k=1, \ldots, \# P_{\iota+1}\right)$ is evaluated and save the minimum distance $c_{p_{j}}$ for each individual $p_{j} \in F_{i}$ (loop for, lines 18 through 20). Then, move the individual $p_{j} \in F_{i}$ whose minimum fitness distance $c_{p_{j}}$ in $F_{i}$ is maximum to $P_{\iota+1}$ (lines 22 through 23). Every time an individual $p$ in $F_{i}$ enters in $P_{\iota+1}$, the values $\left\{c_{p_{j}}, j=1, \ldots, \# F_{i}\right\}$ in $F_{i}$ are reevaluated (lines 24 through 26 ).

The main improvement of Algorithm 1 is that the individuals in each nondominated set $F_{i}$ are sorted using the MaxiMin sorting scheme (line 14) compared with the MaxiMin NSGA-II algorithm in Pires 05. Therefore, good genes are inherited as much as possible by increasing the crossover possibility of good individuals. As a result, the converging speed of optimal solution is largely increased. What is more, Algorithm 1 generates a Pareto-optimal set ${ }^{24}$ instead of one single solution at each generation. And the following decision maker a posterior selects the optimal solution from the set at the end of each generation.

$$
\min _{p_{i} \in P_{\iota+1}} z_{i}=\sum_{j=1}^{n_{o b j}} w_{i j} z_{i j}, i=1, \ldots, N_{p o p}
$$

where $z_{i}$ is the fitness function of each individual $p_{i} \in P_{\iota+1}, w_{i j}$ is the weighting coefficient satisfying $\sum_{j=1}^{n_{o b j}} w_{i j}=1$ and $z_{i j}$ is the normalized $j-t h$ objective function of individual $p_{i}$.

\section{Off-line Motion Planning}

Given EEs' desired positions-orientations $\boldsymbol{X}_{E E}$, the optimal pose $\boldsymbol{\theta}_{o p}$ can be designed using the Algorithm 1. Similarly, given EEs' desired trajectories, the corresponding optimal motion of MDAMS can be designed, but it will be timeconsuming. To this end, an efficient point-to-point bidirectional RRT and gradient decent motion planning Algorithm 2 with a geometric optimization process is proposed in this section to design the motion for MDAMS given the optimal pose. 


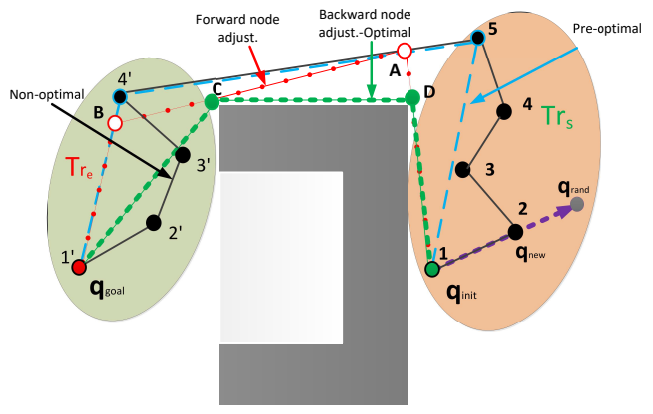

Fig. 3. An example of off-line path planning.

\subsection{Overview}

Throughout Algorithm 2 the path planner maintains two trees $\operatorname{Tr}_{s}$ and $\operatorname{Tr}_{e}$ which root respectively at the initial node $\boldsymbol{q}_{\text {init }}$ and final node $\boldsymbol{q}_{\text {goal }}$ (see Fig. 3). A while loop is activated up till tree $T r_{s}$ and tree $T r_{e}$ meet each other, i.e. DisTree $\leq$ DisMax or there is a direct collision-free connection between $T r_{s}$ and $T r_{e}$. DisTree is the minimum distance between $T r_{s}$ and $T r_{e}, D i s M a x$ is a predefined value.

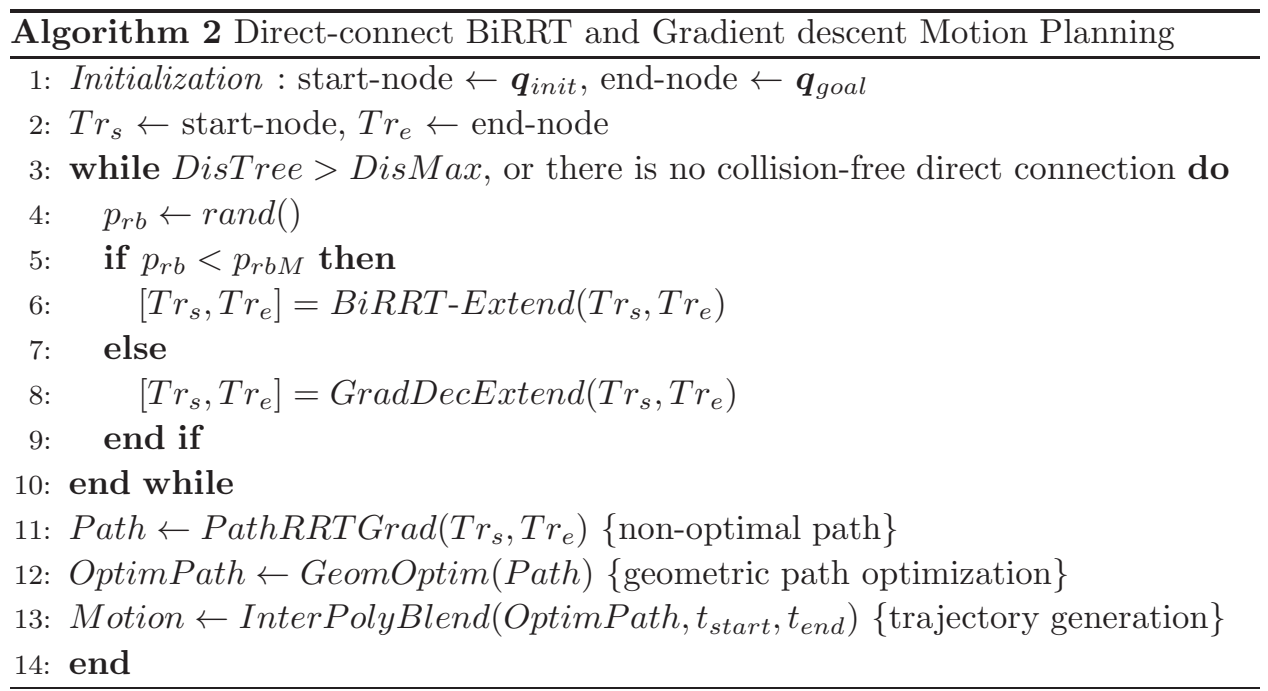

During each while loop, the extending process BiRRT-Extend (line 6) or GradDecExtend (line 8) is selected (lines 3 through 10) to extend $\operatorname{Tr}_{e}$ and $\operatorname{Tr}_{s}$ until two trees meet each other, based on the comparison between a randomly generated value $p_{r b}$ (line 4$)$ and a predefined value $p_{r b M}\left(0 \leq p_{r b M} \leq 1\right)$ which is proportional 
to the clutter of environment. The purple dash line and nodes $\left\{\boldsymbol{q}_{\text {init }}, \boldsymbol{q}_{\text {rand }}, \boldsymbol{q}_{\text {new }}\right\}$ in Fig. 3 illustrate the conventional RRT extending process. ${ }^{8}$ BiRRT-Extend extends two trees at the same time following the above RRT process. GradDecExtend extends two trees directly along the linking line between two trees. In particular, a direct-connect method is proposed even if the two trees $T r_{s}$ and $T r_{e}$ are still very far away from each other. It links $T r_{s}$ and $T r_{e}$ directly (segment $\mathbf{5 - 4 ^ { \prime }}$ ) if there is a collision-free connection between two arbitrary nodes in $T r_{s}$ and $T r_{e}$ respectively. As a result, the path searching process is greatly improved by reducing the sampled nodes.

Process PathRRTGrad (line 11) finds the minimum path from $\boldsymbol{q}_{\text {init }}$ to $\boldsymbol{q}_{\text {goal }}$ by linking two trees $T r_{s}$ and $T r_{e}$ as one single tree that roots at $\boldsymbol{q}_{\text {init }}$ and ends at $\boldsymbol{q}_{\text {goal }}$. Since the obtained path is normally non-optimal, process GeomOptim (line 12 ) is proposed to geometrically optimize the path (see Subsection 4.2). Finally, the process Inter PolyBlend (line 13, see Subsection 4.3) designs a time law to generate the time-specified trajectory within a desired time interval $\left[t_{\text {start }}, t_{\text {end }}\right]$.

\subsection{Geometric path optimization: GeomOptim}

An example of the path planning result is shown in Fig. 3. The black line linking nodes $\left\{\mathbf{1}\left(\boldsymbol{q}_{\text {init }}\right), \ldots, \mathbf{5}, \mathbf{4}^{\prime}, \ldots, \mathbf{1}^{\prime}\left(\boldsymbol{q}_{\text {goal }}\right)\right\}$ represents the designed path after BiRRTExtend and GradDecExtend, which is obviously non-optimal. Therefore, a geometric optimization method which consists of the node rejection and node adjustment processes is proposed. Note that the path after node rejection is pre-optimal, and the path after node adjustment is optimal. The blue, red and green dash lines in Fig. 3 illustrate the proposed method.

\subsubsection{Node rejection}

The node rejection process consists of the following steps: i) initialize the pre-optimal path with $\boldsymbol{q}_{\text {init }}$; ii) test all the nodes along the black line in sequence, and reserve the last node $\mathbf{5}$ which does not interact with obstacles into the pre-optimal path; iii) repeat step ii) until reaching $\boldsymbol{q}_{\text {goal }}$. The blue dash line which consists of nodes $\left\{\boldsymbol{q}_{\text {init }}, \mathbf{5}, \mathbf{4}^{\prime}, \boldsymbol{q}_{\text {goal }}\right\}$ in Fig. 3 is the node rejection result, i.e. the pre-optimal path.

\subsubsection{Node adjustment}

The obtained pre-optimal path after node rejection is usually non-optimal due to the random sampling process. In order to further prune the path, a forward-backward node adjustment process is proposed as follows: i) introduce an auxiliary path and initialize it with $\boldsymbol{q}_{\text {init }}$; ii) test in sequence the segments which link node $\boldsymbol{q}_{\text {init }}$ and the points on segment $5-4^{\prime}$ (the point on segment $5-4^{\prime}$ moves from 5 to $4^{\prime}$ with a fixed step), and reserve the last node $\boldsymbol{A}$ on segment $\mathbf{5}-\mathbf{4}^{\prime}$ which does not collide with obstacles into the auxiliary path; iii) repeat step ii) until reaching $\boldsymbol{q}_{\text {goal }}$, then a path consisting of nodes $\left\{\boldsymbol{q}_{\text {init }}, \boldsymbol{A}, \boldsymbol{B}, \boldsymbol{q}_{\text {goal }}\right\}$ is obtained; iv) flip the obtained path and 
repeat steps i)-iii); v) flip the obtained path again and the optimal path containing nodes $\left\{\boldsymbol{q}_{\text {init }}, \boldsymbol{D}, \boldsymbol{C}, \boldsymbol{q}_{\text {goal }}\right\}$ is obtained. In Fig. 3 the red dash line represents the path after forward node adjustment; the green dash line represents the path after backward node adjustment which is also the optimal path consisting of via-points $\left\{\boldsymbol{q}_{\text {init }}, \boldsymbol{D}, \boldsymbol{C}, \boldsymbol{q}_{\text {goal }}\right\}$.

\subsection{Interpolating linear polynomials with parabolic blends}

The optimal path obtained after line 12 in Algorithm 2 is a geometric path, so a time law is needed. On the other hand, the non-holonomic MDAMS is required to always head forward to show its movement intention to improve human-robot interaction. Therefore, the process InterPolyBlend is used (line 13) to design the smooth trajectory within time interval $\left[t_{\text {start }}, t_{\text {end }}\right]$ using the linear polynomials with parabolic blends interpolation technique ${ }^{53}$. Consider the case where it is required to interpolate $n_{p}$ via-points $\left\{\boldsymbol{q}_{1}, \ldots, \boldsymbol{q}_{n_{p}}\right\}$ at time instants $\left\{t_{i}, i=1, \ldots, n_{p}, t_{1}=\right.$ $\left.t_{\text {start }}, t_{n_{p}}=t_{\text {end }}\right\}$, respectively. The time scope between two adjacent via-points is

$$
\Delta t_{k}=\frac{\operatorname{dis}\left(\boldsymbol{q}_{k}, \boldsymbol{q}_{k+1}\right)}{\sum_{i=1}^{n_{p}-1} \operatorname{dis}\left(\boldsymbol{q}_{i}, \boldsymbol{q}_{i+1}\right)}\left(t_{\text {end }}-t_{\text {start }}\right), k=1, \ldots, n_{p-1} .
$$

In particular, the trajectory generation for the non-holonomic mobile base is described in the following. Suppose that the optimal path of mobile base consists of $n_{p}$ via-points $\boldsymbol{q}_{k}=\left(x_{k}, y_{k}\right)\left(k=1,2, \ldots, n_{p}\right),\left(x_{1}, y_{1}\right)$ and $\left(x_{n_{p}}, y_{n_{p}}\right)$ are respectively the initial and optimal positions, $\phi_{0}$ and $\phi_{d}$ are the initial and optimal orientations, and others are the intermediate ones. The orientation $\phi_{k}$ between two adjacent via-points is calculated as follows:

$$
\phi_{k}=\operatorname{atan}\left(\frac{y_{k+1}-y_{k}}{x_{k+1}-x_{k}}\right), k=1, \ldots, n_{p}-1
$$

to always lead MDAMS head forward. As a result, the orientation list $\left(\phi_{0}, \phi_{1}\right.$, $\left.\ldots, \phi_{n_{p}-1}, \phi_{d}\right)$ is obtained. At each via-point $\boldsymbol{q}_{k}$, the mobile base firstly rotates to the heading orientation $\phi_{k}$, then moves to the next via-point $\boldsymbol{q}_{k+1}$.

\section{On-line Motion Planning}

In the previous section, an efficient off-line motion planning algorithm is presented for MDAMS. However, the designed motion will fail if there are obstacle collisions. Therefore, by extending Algorithm 2, an on-line motion planning Algorithm 3 inspired by Mcleod 16 is introduced in this section under the following assumption. The objective is the motion design given the initial pose and optimal pose $\boldsymbol{\theta}_{o p}$ in dynamic environments for MDAMS.

Assumption 1. The global information of the dynamic environment is known in real time, i.e. the instantaneous positions and geometry characteristics of all obstacles are known, but the obstacles' future movements are unknown. 


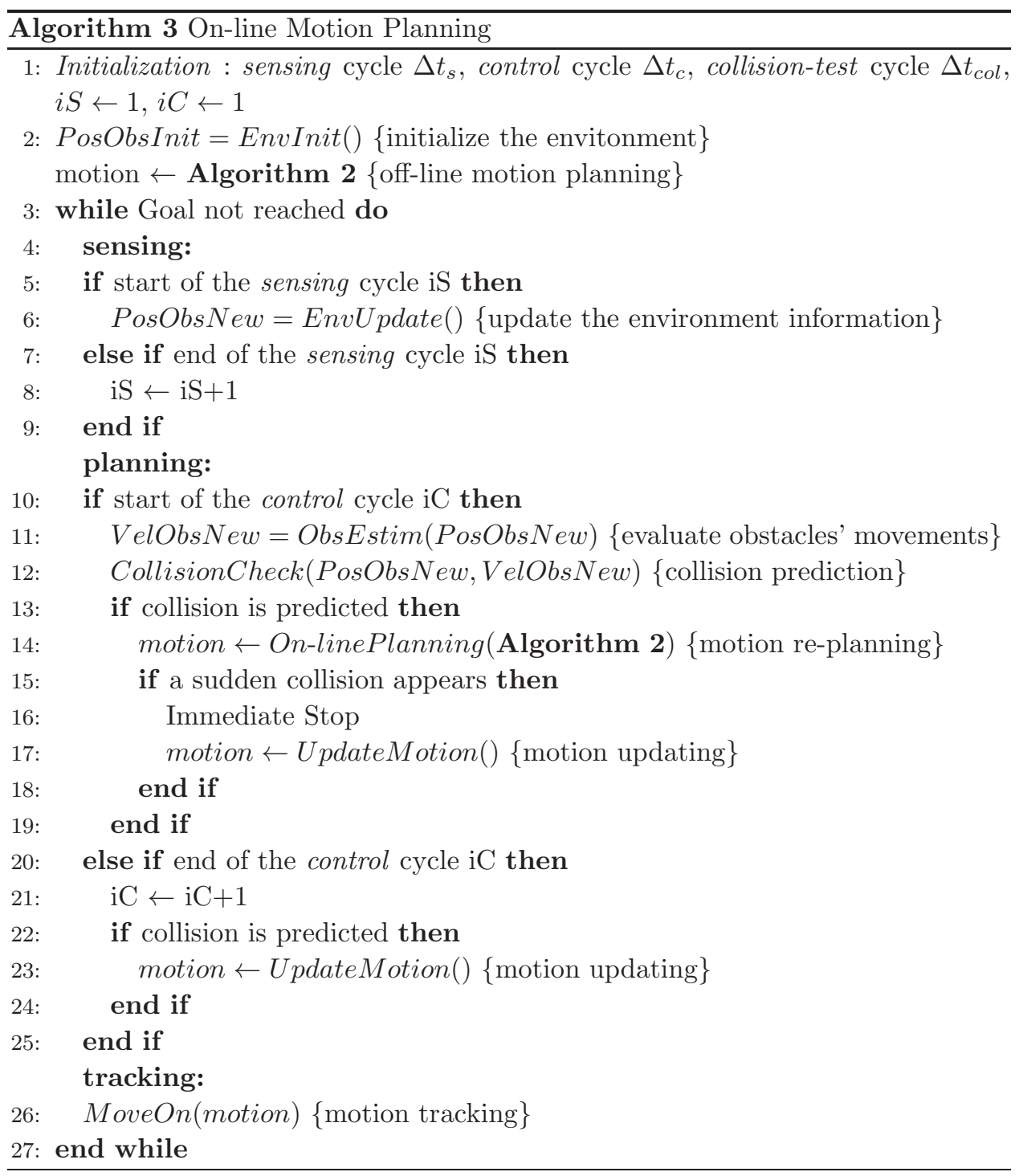

\subsection{On-line motion planning algorithm}

\subsubsection{Overview}

The Algorithm 3 conducts motion planning and execution simultaneously by introducing three cycles: on-line sensing, collision-test and control cycles. In each sensing cycle, changes of the environment are captured and updated. The planning process re-plans the motion according to collision-test's output. In each control cycle, the robot switches to the above re-planned motion if there are predicted collisions.

Algorithm 3 starts by the initialization: the environmental information in- 
cluding positions of all obstacles is captured (EnvInit). An off-line motion is designed using Algorithm 22. The sensing, control and collision-test cycles ( $\left.\Delta t_{s} \leq \Delta t_{c} \leq \Delta t_{c o l}\right)$ and cycle numerations $i S$ and $i C$ are initialized. Then, MDAMS starts to track the designed motion and the on-line motion planning process is activated (while loop, lines 3 through 27).

At the start of each sensing cycle $t_{i S}$, positions of all dynamic obstacles are captured (line 6, EnvUpdate) while conserving the historical ones at the same time.

At the start of each control cycle $t_{i C}$, dynamic obstacles' future positions are predicted (line 11, ObsEstim, see Eqs. (5) and (6)), and the obstacle-collision check is conducted for the current motion (line 12, CollisionCheck). If there are collisions predicted in the collision-test cycle $\left[t_{i C+1}, t_{i C+1}+\Delta t_{c o l}\right]$, the on-line motion replanning process On-linePlanning will be called (line 14).

It is worth mentioning that the start-node of On-linePlanning is $\boldsymbol{q}\left(t_{i C+1}\right)$ of the next control cycle $\left[t_{i C+1}, t_{i C+2}\right]$. The start time $t_{\text {start }}$ of On-linePlanning is $t_{i C+1}$ of the next control cycle $\left[t_{i C+1}, t_{i C+2}\right]$. In particular, if a sudden collision appears (line 15), the MDAMS will stop (line 16) immediately and update the motion (line 17). In that case, the start-node of On-linePlanning will be the current state $\boldsymbol{q}(t)$, and the re-planning start time $t_{\text {start }}$ will be the current time $t$.

At the end of each control cycle $t_{i C+1}$, the motion will be updated (line 23) if collisions are predicted (line 12). As time goes on, the motion tracking is done continuously (line 26, MoveOn).

\subsubsection{Obstacle's motion prediction}

In Assumption 1, we suppose that dynamic obstacles' positions are known in real time. The dynamic obstacles' motions are estimated based on their historical positions (line 11, ObsEstim in Algorithm 3). Specifically, the velocity of dynamic obstacle $i$ is estimated as follows:

$$
\boldsymbol{V}_{o i}=\max \left\{\left\|\left(\boldsymbol{X}_{o i}\left(t_{k}\right)-\boldsymbol{X}_{o i}\left(t_{k-1}\right)\right) / \Delta t_{s}\right\|, k=i S-m_{o}, \ldots, i S\right\}
$$

where $m_{o}$ is the numeration of historical positions of obstacle $i, \boldsymbol{X}_{o i}\left(t_{i}\right)$ is the position of obstacle $i$ at time $t_{i}, \Delta t_{s}$ is the sensing cycle and $i S$ is the sensing cycle numeration. Then, the position of obstacle $i$ at time $t=t_{i C}+\delta t$ can be estimated (line 12, CollisionCheck) as

$$
\boldsymbol{X}_{o i}\left(t_{i C}+\delta t\right)=\boldsymbol{X}_{o i}\left(t_{i C}\right)+\delta t \boldsymbol{V}_{o i}
$$

where $i C$ is the control cycle numeration, $\delta t \in\left(0, \Delta t_{c o l}\right]$.

Recall that in Assumption 1, the global environment information is assumed to be known in real time, which is sometimes difficult to obtain. In that case, the proposed on-line motion planning Algorithm 3 will lose its efficiency. With regard to this, the following relaxed assumption is presented. 
Assumption 2. Only the local environment information within the sensing range is known.

The Algorithm 3 is modified as follows. The environment out of sensing range is treated as a collision-free space. Then, a sequence of via-points $\left\{\boldsymbol{q}_{i}, i=1, \ldots, n_{p}\right\}$ bypassing the sensed obstacles can be found using Algorithm 2 (e.g., four viapoints $\left\{\boldsymbol{q}_{\text {init }}, \boldsymbol{D}, \boldsymbol{C}, \boldsymbol{q}_{\text {goal }}\right\}$ in Fig. (3). At the beginning, the robot tries to move along the first segment $\boldsymbol{q}_{\text {init }}-\boldsymbol{D}$. Then, the environment will be updated as time goes on. This process will repeat until the robot arrives at $\boldsymbol{q}_{\text {goal }}$.

\subsection{EEs' via-point-based MOGA motion planning}

Though the proposed Algorithm 3 can be used to design the motion for any DoF mechanical systems, the collision-free test for a high DoF system is time-consuming and complex. What is worse, the direct motion planning in joint space will lead to unforeseen behaviors in task space, and the via-poses are not considered. Regarding to this, the objective of this subsection is to propose an EEs' via-point-based MOGA motion planning algorithm to optimize the via-poses given EEs' desired positionsorientations.

\subsubsection{Algorithm}

The mobile base's trajectory $\boldsymbol{\theta}_{m}=(x, y, \phi)$ and the joint trajectory of manipulator $\left(\boldsymbol{\theta}_{w}, \boldsymbol{\theta}_{R}, \boldsymbol{\theta}_{L}\right)$ characterize the MDAMS's motion (see Section 2). Recall that the initial pose and EEs' desired positions-orientations are known in Section 2 . A sequence of collision-free via-points $\left\{\boldsymbol{X}_{i}=\left[\boldsymbol{X}_{R_{i}}^{T} \boldsymbol{X}_{L_{i}}^{T}\right]^{T} \in \mathbb{R}^{4 m \times 1}, i=1, \ldots, n_{p}\right\}$ are designed using Algorithm 3, where $\boldsymbol{X}_{R_{i}}$ and $\boldsymbol{X}_{L_{i}}$ are EEs' via-points, and $n_{p}$ is the number of EEs' via-points. The objective is to design optimal via-poses $\boldsymbol{\Theta}_{i}=\left(x_{i}, y_{i}, \phi_{i}, \boldsymbol{\theta}_{w_{i}}, \boldsymbol{\theta}_{R_{i}}, \boldsymbol{\theta}_{L_{i}}\right)$ corresponding to $\left\{\boldsymbol{X}_{i}\right\}$.

MOGAs (e.g. the improved MaxiMin NSGA-II algorithm 1) are used to search for the optimal via-poses. The chromosome is chosen as $\left\{\boldsymbol{\Theta}_{1}, \ldots, \boldsymbol{\Theta}_{n_{p}}\right\}$. The searching interval for each via-pose is $\left\{\boldsymbol{\Theta}_{i j} \in\left[\boldsymbol{\theta}_{j \min }, \boldsymbol{\theta}_{j \max }\right], j=1, \ldots, n\right\}$, where $\boldsymbol{\theta}_{j_{\max }}$ and $\boldsymbol{\theta}_{j_{\min }}$ are respectively the upper and lower boundaries of $\boldsymbol{\theta}_{j}$ (see Section 2). Specifically, $\left(x_{i}, y_{i}\right)$ is searched within the reaching region of via-point $\left\{\boldsymbol{X}_{i}\right\}$, and $\phi_{i}$ is searched within $[-\pi, \pi]$. The via-pose-based objective functions are defined in the next subsection.

\subsubsection{Via-pose-based objective functions}

- EE's positioning accuracy: The via-poses $\left\{\boldsymbol{\Theta}_{i}\right\}$ are required to bypass all the EE's via-points $\left\{\boldsymbol{X}_{i}\right\}$. Hence, the first objective function is defined as follows: $f_{a}(\boldsymbol{\Theta})=\sum_{i=1}^{n_{p}}\left\|\boldsymbol{X}_{i}-\boldsymbol{X}_{i}\left(\boldsymbol{\Theta}_{i}\right)\right\|$, where $\boldsymbol{X}_{i}\left(\boldsymbol{\Theta}_{i}\right)$ is the EEs' via-points corresponding to via-pose $\boldsymbol{\Theta}_{i}$ based on the forward kinematics expressed by $T_{R}$ and $T_{L}$ in Section 2 
- Joint displacement: The least joint displacement is required to minimize energy consumption. Then, $f_{b}(\boldsymbol{\Theta})=\sum_{i=1}^{n_{p}} \sum_{j=1}^{n}\left\|\left(\boldsymbol{\Theta}_{i j}-\boldsymbol{\Theta}_{(i-1) j}\right) /\left(\boldsymbol{\theta}_{j_{\max }}-\boldsymbol{\theta}_{j_{\min }}\right)\right\|$.

- Collision evaluation: In order to take into account the robot-obstacle intersection, simplify the MDAMS as a skeleton in Fig. 1(b) and introduce eight control points $(1, \ldots, 8)$ and eight links $(a, \ldots, h)$, i.e. the CoMs of mobile base, waist, two shoulders, two elbows and two wrists, and eight connection links between them. Then, define the third objective function as follows:

$f_{c}(\boldsymbol{\Theta})=\sum_{i=1}^{n_{p}} \sum_{j=1}^{n_{c}} L c_{i j}\left(\boldsymbol{\Theta}_{\boldsymbol{i}}\right) / L$, where $n_{c}$ is the number of links on MDAMS, $L c_{i j}\left(\boldsymbol{\Theta}_{\boldsymbol{i}}\right)$ is the length of link which collides with obstacles along the link $j$, and $L$ is the length of eight defined links. Take the situation in Fig. 1 (b) as an example: the left forearm intersects an obstacle, then the third objective function is given as $f_{c}=h /(a+b+\cdots+h)$ for one via-pose, $n_{p}=1$.

- Directional manipulability: To facilitate the motion bypassing all the EEs' via-points $\left\{\boldsymbol{X}_{i}\right\}$, there is a directional manipulability preference between two adjacent via-points $\boldsymbol{X}_{i}$ and $\boldsymbol{X}_{i+1}$ in task space. Then,

$f_{d}(\boldsymbol{\Theta})=-\sum_{i=1}^{n_{p}-1}\left(\Omega_{B d i r}\left(\boldsymbol{d}_{R_{i}}\right)+\Omega_{B d i r}\left(\boldsymbol{d}_{L_{i}}\right)\right)$, where $\Omega_{B d i r}\left(\boldsymbol{d}_{R_{i}}\right)$ and $\Omega_{B d i r}\left(\boldsymbol{d}_{L_{i}}\right)$ are respectively the directional manipulabilities of right and left EEs which are defined as follows:

$$
\Omega_{B d i r}\left(\boldsymbol{d}_{\kappa_{i}}\right)=\sum_{k=1}^{m}\left\|\left(\boldsymbol{d}_{\kappa_{i}}^{T} \cdot \boldsymbol{u}_{i k}\right) \sigma_{i k}\right\|
$$

where $\boldsymbol{d}_{\kappa_{i}}=\left(x_{\kappa}\left(\boldsymbol{\Theta}_{i+1}\right)-x_{\kappa}\left(\boldsymbol{\Theta}_{i}\right)\right) /\left\|x_{\kappa}\left(\boldsymbol{\Theta}_{i+1}\right)-x_{\kappa}\left(\mathbf{\Theta}_{i}\right)\right\|$ is the unit vector along each segment $\left\{\boldsymbol{X}_{i}\left(\boldsymbol{\Theta}_{i}\right)-\boldsymbol{X}_{i+1}\left(\boldsymbol{\Theta}_{i+1}\right), i=1, \ldots, n_{p}-1\right\}$. (.) is the dot product and $x_{\kappa}, \kappa \in\{R, L\}$ is defined in Section 2] Decompose Jacobian $J_{\kappa}\left(\boldsymbol{\Theta}_{i}\right) \in \mathbb{R}^{m \times n}, \kappa \in$ $\{R, L\}$ in Section 2 using the singular value decomposition technique $J_{\kappa}\left(\boldsymbol{\Theta}_{i}\right)=$ $U_{i} \Sigma_{i} V_{i}, \boldsymbol{u}_{i k}$ is the $k$-th column vector of $U_{i} \in \mathbb{R}^{m \times m}, \sigma_{i k}$ is the $k$-th singular value of $\Sigma_{i} \in \mathbb{R}^{m \times n}$ and $V_{i} \in \mathbb{R}^{n \times n}$.

As a result, the optimization problem is formulated as follows:

$$
\begin{gathered}
\min \left(f_{a}(\boldsymbol{\Theta}), f_{b}(\boldsymbol{\Theta}), f_{c}(\boldsymbol{\Theta}), f_{d}(\boldsymbol{\Theta})\right) . \\
\text { s.t. } \boldsymbol{\Theta} \in C_{\text {free }}
\end{gathered}
$$

\section{Simulations Results}

\subsection{Optimal pose design and path planning}

In Fig. 4. a table is surrounded by four chairs $A, B, C$ and $D$. The task is to design the motion for MDAMS to manipulate chair $A$, which can be divided into two subtasks: the optimal pose $\boldsymbol{\theta}_{o p}$ design around chair $A$ and the collision-free approaching motion design. The EEs' desired positions are known as $x_{R d}=(2.7,0.3,0)$, $x_{L d}=(2.7,-0.3,0)$, and the initial positions are $x_{R}=(-0.2,0.25,-0.219), x_{L}=$ $(-0.2,0.55,-0.219)$. Based on the robot's dimension in Table 1 , the expected position and orientation of mobile base are calculated as $\left(x_{m e}, y_{m e}\right)=(3,0)$ and $\phi_{d e}=$ $180^{\circ}$, respectively. Set the initial pose of MDAMS as $\boldsymbol{\theta}_{\text {init }}=\left(-0.2,0.4,0, \mathbf{0}_{16}\right)$. 
Table 2. The designed optimal pose $\boldsymbol{\theta}_{o p}[\mathrm{~m}, \mathrm{deg}]$.

\begin{tabular}{c|r|r|r|r|r|r|r}
\hline $\boldsymbol{\theta}_{m} \& \boldsymbol{\theta}_{w}$ & 2.95 & 0.027 & 166.77 & 5.90 & 0.24 & - & - \\
\hline $\boldsymbol{\theta}_{R}$ & 1.11 & 13.73 & 33.19 & 74.63 & 3.85 & 49.38 & 4.81 \\
\hline $\boldsymbol{\theta}_{L}$ & 2.77 & 22.07 & 8.19 & 64.46 & 67.65 & 67.63 & 23.91 \\
\hline
\end{tabular}
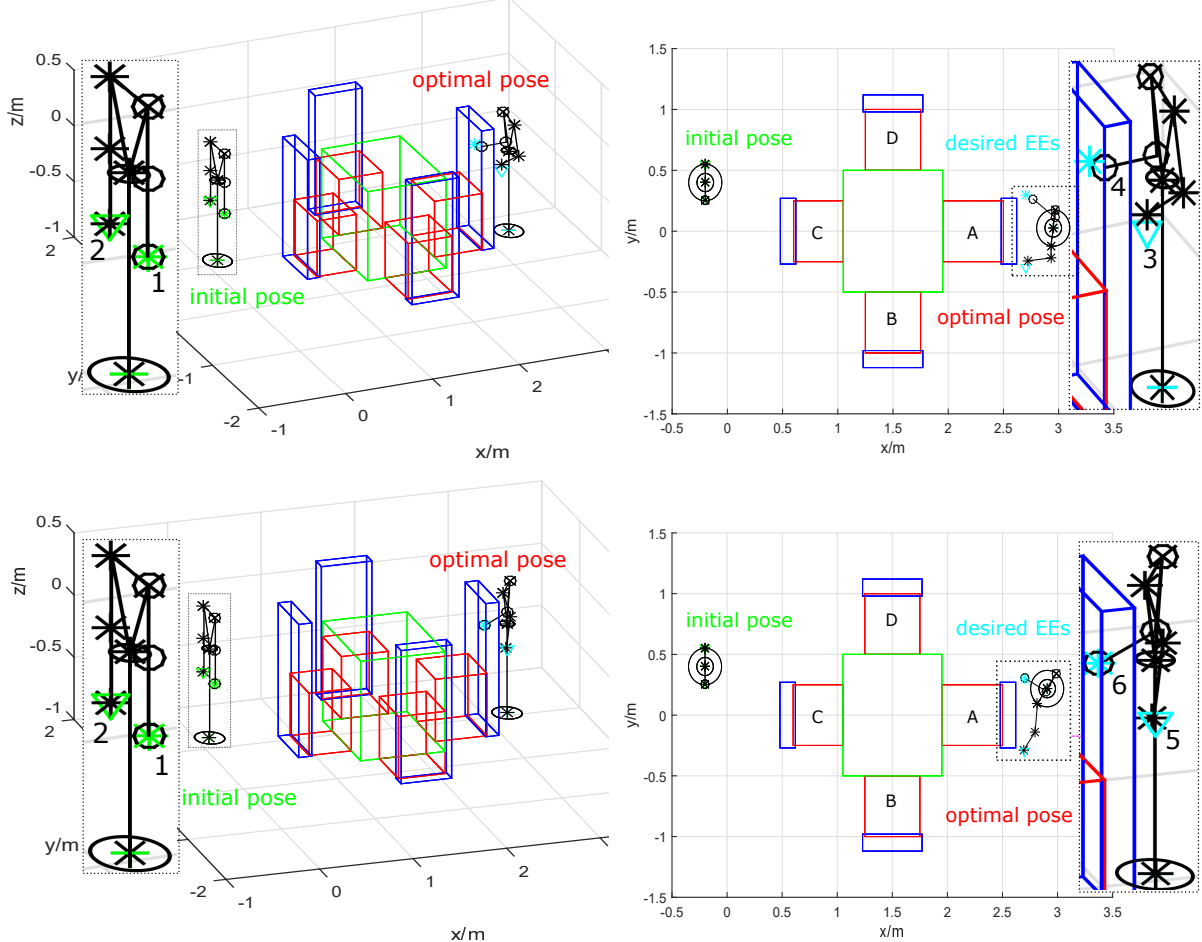

Fig. 4. Pose design of MDAMS to manipulate chair A. Up: improved MaxiMin NSGA-II algorithm. Down: combined fitness function-based MOGA.

\subsubsection{Optimal pose design}

This subsection validates the Algorithm 1 Set the population size as $N_{\text {pop }}=100$, the generation number as $N_{G}=120$ and the genetic crossover and mutation probabilities as $p_{c}=0.7$ and $p_{m}=0.3$. According to the importance of each objective, the coefficients in Eq. (2) are chosen as $w_{i}=(0.54,0,0.04,0.02,0.4), i=1, \ldots, N_{\text {pop }}$. After $N_{G}$ generations, the designed optimal pose $\boldsymbol{\theta}_{o p}$ is shown in Table 2 and Fig. 4.

It can be seen that the designed optimal position of mobile base is $\left(x_{m d}, y_{m d}\right)=$ $(2.95,0.027)$ which is near the expected position $\left(x_{m e}, y_{m e}\right)$, the positioning error is $(-0.05,0.027)$; the designed optimal orientation of mobile base is $\phi_{d}=166.77^{\circ}$ 


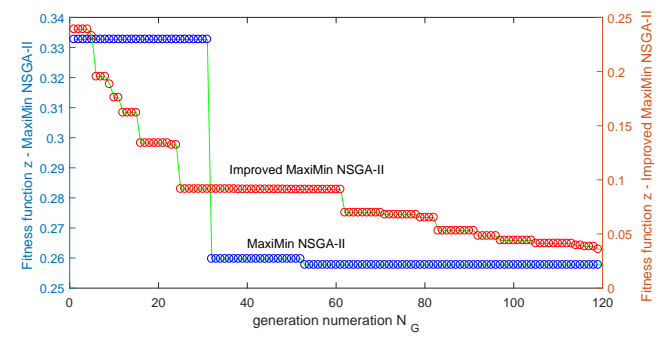

Fig. 5. Comparison between the improved MaxiMin NSGA-II and MaxiMin NSGA-II algorithms.

which is near the expected orientation $\phi_{d e}$, the orientation error is 0.23 rad.

The up two figures in Fig. 4 show the motion planning results using the proposed improved MaxiMin NSGA-II Algorithm [1. The black skeletons represent the designed MDAMS. Points 1 and 2 show the initial positions of right and left EEs. The cyan plus shows the designed optimal position $\left(x_{m d}, y_{m d}\right)$ of mobile base. Points 3 and 4 show the designed positions $\left(x_{R}\left(\boldsymbol{\theta}_{o p}\right), x_{L}\left(\boldsymbol{\theta}_{o p}\right)\right)$ of right and left EEs which can be calculated according to the forward kinematics. It can be seen that the designed optimal pose $\boldsymbol{\theta}_{o p}$ is "human-like" with good positioning accuracy to EEs' desired positions $\left(x_{R d}, x_{L d}\right)$ in the objective function $f_{1}$ of Subsection 3.1 . As a comparison, the motion planning results using the combined fitness functionbased MOGA are shown in the down two figures in Fig. 4 with the combined fitness function being defined in Eq. (2). It can be seen that even though the EEs' positioning accuracy is better than that of the Algorithm 1] the designed optimal mobile base's position-orientation and manipulator's configuration are twisted, not "human-like" and far away from expectations.

Furthermore, two optimal pose design simulations are realized in Fig. 5 which shows that the optimal pose's fitness function value evolution of Eq. (2). The optimal solution converges more quickly and continuously with better performance using the Algorithm 1.

\subsubsection{Path planning and optimization for the mobile base}

This subsection validates the path planning Algorithm 2. The mobile base has a circle geometry. To guarantee the collision-free navigation for the mobile base from the initial position to the designed optimal position $\left(x_{m d}, y_{m d}\right)$, we enlarge the obstacles by a security hull $\delta_{s m}=0.3 \mathrm{~m}$ including the mobile base's dimension. The Fig. 6 (a) shows the designed path without geometric optimization. The blue star points, blue plus points and the blue line represent the sampling nodes of the start exploring tree $T r_{s}$, the end exploring tree $T r_{e}$ and the designed path, respectively. It can be seen that two exploring trees are connected directly once there is a collision-free connection between them even if they are still very far away 
(a)

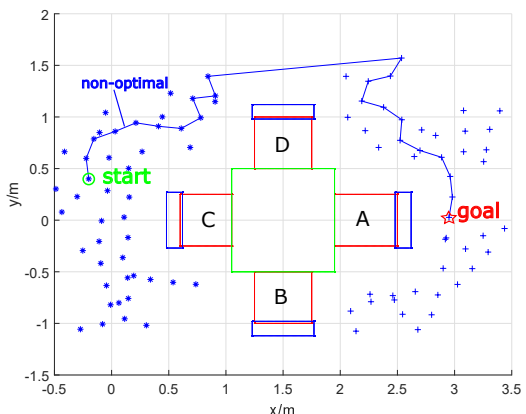

Fig. 6. Path planning results for the mobile base: (a) without geometric optimization, (b) with geometric optimization.

from each other. A collision-free approaching path is designed linking the mobile base's initial and desired positions, however, it is not the optimal path.

The Fig. 6(b) shows the path optimization results using the proposed geometric optimization method. The red line represents the pre-optimal path after node rejection, the green line represents the optimal path after node adjustment which is also the designed optimal path. In the optimal path, there are in total $n_{p}=5$ via-points which can be adjusted within their neighborhoods to increase the clearance.

\subsection{On-line motion planning for the mobile base}

This subsection is to verify the Algorithm 3 . Note that motion tracking of the mobile base is not considered in this paper. The dynamic obstacles move on the ground with bounded stochastic velocities. Set the sensing, control and collision-test cycles as $\Delta t_{s}=0.5 \mathrm{~s}, \Delta t_{c}=1 \mathrm{~s}$ and $\Delta t_{c o l}=2 \mathrm{~s}$. In the following, on-line motion planning for the mobile base is realized using Matlab in a standard PC (Intel(R) Core(TM) i5-3317U CPU @ 1.70GHz 1.70 GHz, 4,00Go, x64). Suppose that the mobile base locates initially at $(4.5,1)$ and its desired position is $(1,6)$. Figs. 7 and 8 show the motion planning results. In detail, Fig. (7)(a) shows the off-line motion planning results using Algorithm 2. The blue star points and line represent the non-optimal path. The red line represents the path after node rejection, and the green line represents the optimal path after node adjustment. The black dot line represents the mobile base's trajectory using the linear polynomials with parabolic blends interpolation technique InterPolyBlend. We can see that the trajectory coincides with the optimal path. As a comparison, the magenta line represents the planned trajectory using B-spline interpolation which generates too many orientations for the mobile base and may bring unforeseen collisions.

The star magenta points from Fig. 7 (b) to Fig. 8 (f) represent the movements of mobile base. The black line behind the magenta points represents the mobile base's historical positions, and the black line ahead the magenta points represents 

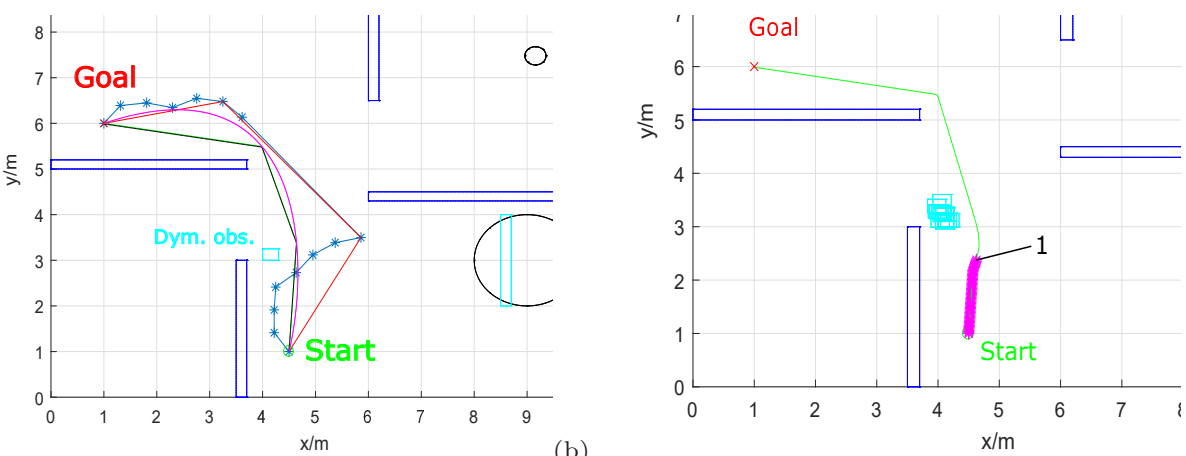

(a)

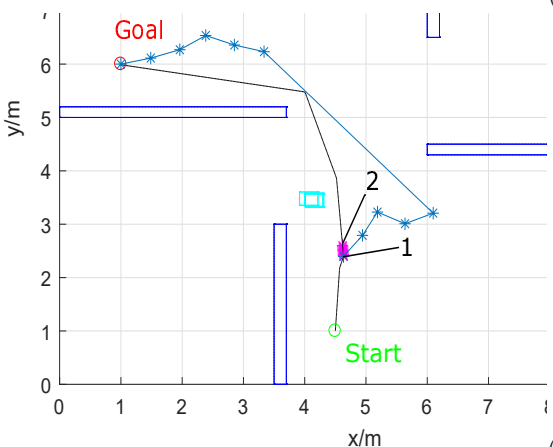

(c) (b)

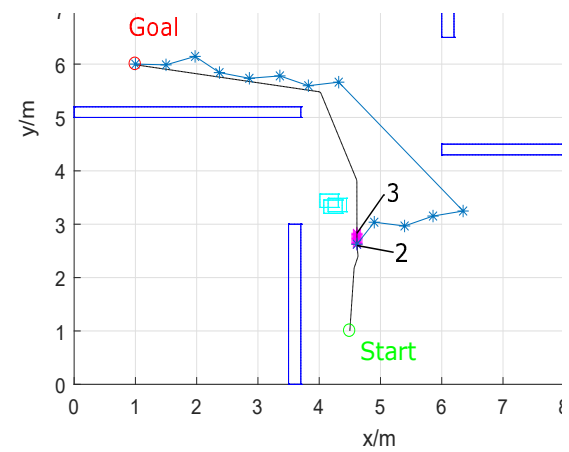

Fig. 7. On-line motion planning results for mobile base (I). (a) Off-line motion planning results, (b) Phase 1, (c) Phase 2, (d) Phase 3.

(a)
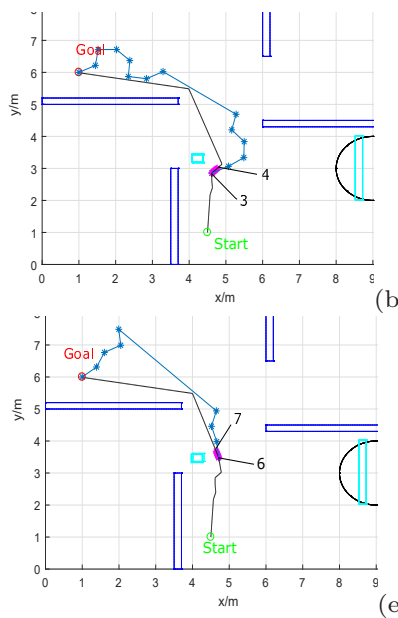

(b)
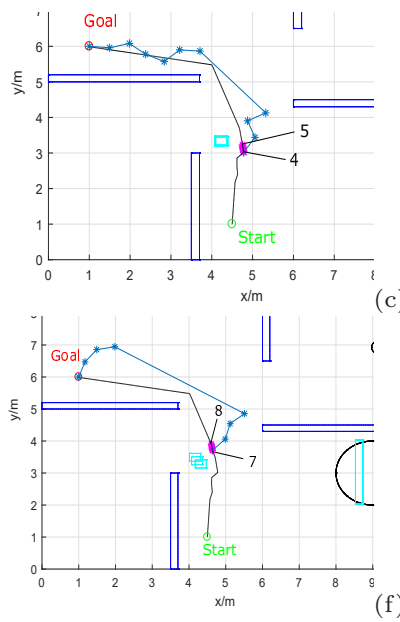

(c)
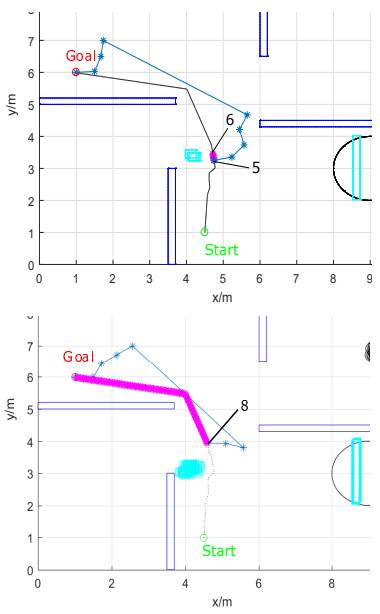

Fig. 8. On-line motion planning results for mobile base (II). (a) Phase 4, (b) Phase 5, (c) Phase 6, (d) Phase 7, (e) Phase 8, (f) Phase 9. 
the designed motion by Algorithm 3 in real time. It can be seen that each time there are predicted collisions. The on-line planning process is activated to design the new motion for mobile base. Throughout this simulation, there are in total 8 On-linePlanning calls, which are illustrated by the blue star points and lines from phase 2 to phase 9 , to update the motion to avoid the cyan cuboid dynamic obstacle on the way to the desired position. Fig. 8 (f) shows that the motion remains the same after bypassing the dynamic cyan cuboid (see phase 9 ).

\subsection{Motion planning for MDAMS}

Theoretically speaking, Algorithm 3 can be used to design the motion for any DoF mechanical systems, but the computational cost will increase and the via-poses will be difficult to predict. This subsection is to verify the EEs' via-point-based MOGA motion planning algorithm.

Suppose that the initial pose of MDAMS is $\boldsymbol{\theta}_{0}=\left(7,1,0, \mathbf{0}_{16}\right)$; the left EE's initial and desired positions are $(7,1.15,0.612)$ (green circle) and $(8.1,1.3,0.66)$ (see Fig. 9 (a)), respectively over a $2 m \times 1 m \times 0.6 m$ table (cyan cuboid) at $(9.5,1,0)$; the right EE's initial and desired positions are $(7,0.85,0.612)$ (blue star) and $(8.1,0.7,0.66)$

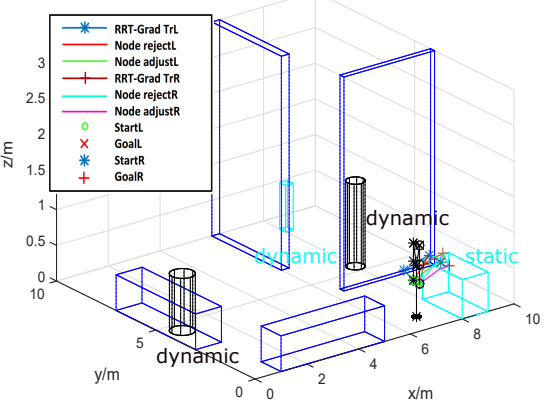

(a)

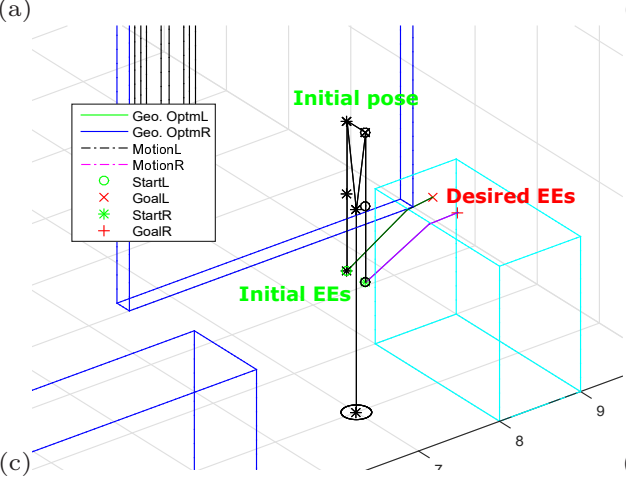

(b)
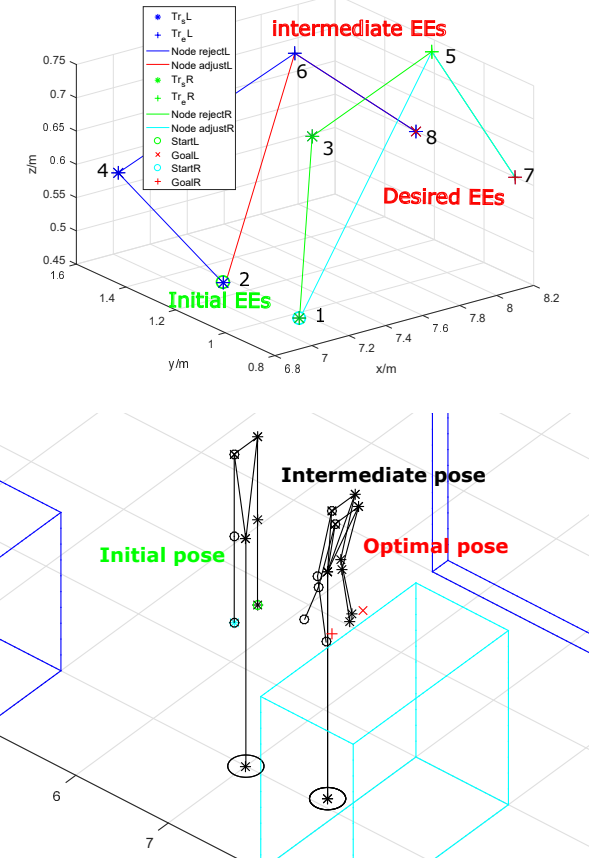

Fig. 9. Motion planning results for MDAMS. (a) Path planning (global view) for EEs, (b) Path planning (local view) and via-points for EEs, (c) Trajectory planning for EEs, (d) Optimal viaposes for MDAMS. 
(red plus), respectively. The objective is to reach EEs' desired positions among the obstacles with reasonable via-poses. The motion planning results are shown in Fig. 9.

Firstly, the EEs' via-points are designed using Algorithm 3 Fig. 9 (a) shows the path planning results for two EEs, Fig. 9 (b) zooms in the results. There are in total three pairs of via-points, i.e. $1-2,5-6$ and $7-8$ in the optimal path. The pair 3-4 is rejected after node adjustment. Fig. 9(c) shows EEs' trajectories bypassing $1-2,5-6$ and $7-8$. However, the movements of mobile base and other joints are not decided. To this end, the EEs' via-point-based MOGA algorithm is used to optimize the via-poses of the whole MDAMS. Fig. 9 (d) shows the designed optimal via-poses corresponding to $1-2,5-6$ and $7-8$. It can be seen that the designed via-poses avoid the table and have the cognitive shock-free "human-like" behaviors.

\section{Discussion}

The main contributions of this paper are "human-like" motion planning algorithms to improve the quality of human-robot interaction. They avoid calculating inverse Jacobian and satisfy multiple constraints simultaneously.

The Algorithm 1 is used to design simultaneously the mobile base's positionorientation and the manipulator's configuration. Other constraints found in the literature can be also integrated into Algorithm 11, and it can be used for other multi-objective optimization problems. Theoretically speaking, Algorithm 1 can be used to design MDAMS's motion given the desired trajectories of EEs, but the shortcoming is that it is time-consuming. So it is not applicable for on-line motion planning. To this end, an efficient pose-to-pose bidirectional RRT and gradient decent motion planning algorithm with a geometric optimization method is proposed to design the motion from the initial pose to the optimal pose.

Berenson ${ }^{14,55}$ proved the probabilistic completeness of RRT-based algorithms under end-effector's orientation constraints. In this paper, the volume of the manifold in an embedding space is not zero, thus the probability of generating a path on the manifold of the proposed Algorithm 2 by rejection sampling in the embedding space will go to 1 as the number of samples goes to infinity.

Geraerts ${ }^{34}$ pointed out that many motion planning techniques generate low quality paths, and presented a number of techniques to improve the quality of paths. However, complex geometric calculation of the medial axis is required and the effectiveness for articulated robots remains to be studied. The same problem happened in Wilmarth 89. In this paper, only the planned via-points are needed to be adjusted within their neighborhoods to increase the clearance without calculating the medial axis. Compared with Bakdi 17, the proposed geometric optimization method is simpler and more efficient without considering different cases in detail. Moreover, different from Greiff 17 which uses a projection process to find the collision-free viapoints, the Algorithm 3 in this paper can easily find them. The simple geometric 
optimization method can be applied to other planning methods like roadmaps or RRTs for path pruning. Different from the method in Bakdi 17 which searches for a great number of via-poses from the initial pose to the optimal pose, the via-pointbased MOGA algorithm in this paper only needs to design a few via-poses to achieve "human-like" collision-free behaviors.

\section{Conclusion}

The motion planning for a humanoid MDAMS is researched in this paper. The improved MaxiMin NSGA-II algorithm is proposed to design the optimal pose by optimizing five objective functions. Besides, an efficient direct-connect bidirectional RRT and gradient descent method is proposed to speed up greatly the sampling process. And a geometric optimization method is designed to always guarantee the consistent and shortest collision-free path. "Natural" head forward behaviors are realized to "tell" motion intentions by assigning reasonable mobile base's orientations. By designing on-line sensing, collision-test and control cycles, motion planning in dynamic environments with unknown obstacles is achieved. Furthermore, an EEs' via-point-based MOGA motion planning algorithm is proposed to optimize the via-poses. The proposed algorithms in this paper can be used for motion planning for various robots. In order to implement the proposed motion methods and to improve the long-term performance of MDAMS, the future work will focus on validation of the proposed algorithms on virtual prototype. Besides, uncertainties come from environments, sensors and computation will be taken into account for real applications.

\section{References}

1. H. S. Yang et al., Design and development of biped humanoid robot, AMI2, for social interaction with humans, in IEEE-RAS Int. Conf. Humanoid Robots (IEEE Press, Genova, Italy, 2006), pp. 352-357.

2. J. Lafaye, D. Gouaillier, P. B. Wieber, Linear model predictive control of the locomotion of Pepper, a humanoid robot with omnidirectional wheels, in IEEE-RAS Int. Conf. Humanoid Robots (IEEE Press, Madrid, Spain, 2014), pp. 336-341.

3. M. A. Diftler et al., Robonaut 2 - The first humanoid robot in space, in IEEE Int. Conf. Robotics and Automation (ICRA), 2011, pp. 2178-2183.

4. Low, S. C., Phee, L., A review of masterslave robotic systems for surgery, International Journal of Humanoid Robotics (INT J HUM ROBOT) 3(04) (2006), 547-567.

5. S. Kuindersma et al., Optimization-based locomotion planning, estimation, and control design for the atlas humanoid robot, in Autonomous Robot 40(3) (2016) 429-455.

6. B. Cohen, S. Chitta, M. Likhachev, Search-based planning for dual-arm manipulation with upright orientation constraints, in IEEE Int. Conf. Robotics and Automation (ICRA)(IEEE Press, St. Paul, MN, USA, 2012), pp. 3784-3790.

7. L. E. Kavraki et al., Probabilistic roadmaps for path planning in high-dimensional configuration spaces, IEEE Trans. Robot. Autom. 12(4) (1996) 566-580.

8. LaValle, Steven M., Rapidly-exploring random trees: A new tool for path planning[J]. 1998.

9. M. H. Korayem, S. R. Nekoo, The SDRE control of mobile base cooperative manipu- 
lators: Collision free path planning and moving obstacle avoidance, Robotics and Autonomous Systems 86 (2016) 86-105.

10. R. Raja, A. Dutta, Path planning in dynamic environment for a rover using $\mathrm{A}^{*}$ and potential field method, in Int. Conf. Advanced Robotics (ICAR)(Hong Kong, China, 2017), pp. 578-582.

11. Y. C. Tsai, H. P. Huang, Motion planning of a dual-arm mobile robot in the configuration-time space, in IEEE/RSJ Int. Conf. Intelligent Robots and Systems $(I R O S)$ (St. Louis (MO), USA, 2009), pp. 2458-2463.

12. C. L. Lewis, Trajectory generation for two robots cooperating to perform a task, in IEEE Int. Conf. Robotics and Automation(ICRA) (IEEE Press, Minnesota, USA, 1996), vol.2., pp. 1626-1631.

13. J. P. Saut et al., Planning pick-and-place tasks with two-hand regrasping, in IEEE/RSJ Int. Conf. Intelligent Robots and Systems (IROS)(IEEE Press, Taipei, Taiwan, 2010), pp. 4528-4533.

14. D. Berenson, S. S. Srinivasaz, Probabilistically complete planning with end-effector pose constraints, in IEEE Int. Conf. Robotics and Automation (ICRA)(IEEE Press, Alaska, USA, 2010), pp. 2724-2730.

15. P. Michel et al., Motion planning using predicted perceptive capability, International Journal of Humanoid Robotics, 6(03) (2009), 435-457.

16. M. H. Korayem, M. Nazemizadeh, V. Azimirad, Optimal trajectory planning of wheeled mobile manipulators in cluttered environments using potential functions, Scientia Iranica 18(5) (2011) 1138-1147.

17. M. H. Korayem, V. Azimirad, M. I. Rahagi, Maximum Allowable Load of Mobile Manipulator in the Presence of Obstacle Using Non-Linear Open and Closed Loop Optimal Control, Arabian Journal for Science and Engineering (AJSE) 39(5) (2014) 4103-4117.

18. K. Harada et al., Base position planning for dual-arm mobile manipulators performing a sequence of pick-and-place tasks, in IEEE-RAS 15th Int. Conf. Humanoid Robots (Humanoids) (IEEE Press, Seoul, South Korea, 2015), pp. 194-201.

19. M. Galicki, Real-time constrained trajectory generation of mobile manipulators, Robotics and Autonomous Systems 78 (2016) 49-62.

20. K. Deb, Multi-objective optimization using evolutionary algorithms, vol.16, (NY, USA, John Wiley \& Sons, 2001).

21. T. T. Mac et al., Heuristic approaches in robot path planning: A survey, Robotics and Autonomous Systems 86(Supplement C) (2016) 13-28.

22. J. K. Parker, A. R. Khoogar, D. E. Goldberg, Inverse kinematics of redundant robots using genetic algorithms, in Int. Conf. Robotics and Automation (ICRA)(IEEE Press, Arizona, USA, 1989), pp. 271-276 vol.1.

23. M. Zhao, N. Ansari, E. S. H. Hou, Mobile Manipulator Path Planning By A Genetic algorithm, in Proceedings of the IEEE/RSJ Int. Conf. Intelligent Robots and Systems (IROS'92)(North Carolina, USA, 1992), vol.1, pp. 681-688.

24. M. d. G. Marcos, J. A. Tenreiro Machado, T. P. Azevedo-Perdicolis, A multi-objective approach for the motion planning of redundant manipulators, Applied Soft Computing 12(2) (2012) 589-599.

25. A. Bakdi et al., Optimal path planning and execution for mobile robots using genetic algorithm and adaptive fuzzy-logic control, Robotics and Autonomous Systems 89 (2017) 95-109.

26. D. E. Goldberg, Genetic Algorithms in Search, Optimization and Machine Learning, 1st edn. (Boston, MA, USA, Addison-Wesley Longman Publishing Co., Inc., 1989).

27. N. Srinivas, K. Deb, Muiltiobjective Optimization Using Nondominated Sorting in 
Genetic Algorithms, Evolutionary Computation 2 (1994) 221-248.

28. K. Deb et al., A fast and elitist multiobjective genetic algorithm: NSGA-II, IEEE Trans. Evol. Comput. 6(2) (2002) 182-197.

29. E. J. S. Pires, P. B. d. M. Oliveira, J. A. T. Machado, Multi-objective MaxiMin Sorting Scheme, in International Conference on Evolutionary Multi-Criterion Optimization (Springer, Berlin, Heidelberg, 2005), pp. 165-175.

30. Y. Choi, H. Jimenez, D. N. Mavris, Two-layer obstacle collision avoidance with machine learning for more energy-effcient unmanned aircraft trajectories, Robotics and Autonomous Systems 98(Supplement C) (2017) 158-173.

31. O. Adiyatov, H. A. Varol, A novel RRT*-based algorithm for motion planning in Dynamic environments, in IEEE Int. Conf. Mechatronics and Automation (ICMA)(Takamatsu, Japan, 2017), pp. 1416-1421.

32. S. Primatesta, L. O. Russo, B. Bona, Dynamic trajectory planning for mobile robot navigation in crowded environments, in IEEE 21st Int. Conf. Emerging Technologies and Factory Automation (ETFA)(Berlin, Germany, 2016), pp. 1-8.

33. S. A. Wilmarth, N. M. Amato, P. F. Stiller, MAPRM: a probabilistic roadmap planner with sampling on the medial axis of the free space, in Proceedings 1999 IEEE Int. Conf. Robotics and Automation (ICRA), pp. 1024-1031 vol.2.

34. R. Geraerts, M. H. Overmars, Clearance based path optimization for motion planning, in IEEE Int. Conf. Robotics and Automation (ICRA)(LA, USA,2004), pp. 2386-2392 Vol.3.

35. T. Mercy et al., Spline-Based Motion Planning for Autonomous Guided Vehicles in a Dynamic Environment, IEEE Trans. Control Syst. Technol. (2017) pp. 1-8.

36. S. Lee, H. Moradi, C. Yi, A real-time dual-arm collision avoidance algorithm for assembly, in IEEE International Symposium on Assembly and Task Planning (ISATP)(California, USA, 1997), pp. 7-12.

37. J. P. van den Berg, M. H. Overmars, Roadmap-based motion planning in dynamic environments, IEEE Trans. Robot. 21(5) (2005) 885-897.

38. G. Pajak, I. Pajak, Motion planning for mobile surgery assistant, Acta Bioeng Biomech 16(2) (2014) 11-20.

39. Y. Yang, V. Ivan, S. Vijayakumar, Real-time motion adaptation using relative distance space representation, in 2015 Int. Conf. Advanced Robotics (ICAR), pp. 21-27.

40. G. Chen et al., A novel autonomous obstacle avoidance path planning method for manipulator in joint space, in 2014 IEEE Conference on Industrial Electronics and Applications, pp. 1877-1882.

41. G. Xin et al., Real-time dynamic system to path tracking and collision avoidance for redundant robotic arms, The Journal of China Universities of Posts and Telecommunications 23(1) (2016) 73-96.

42. D. Han, H. Nie, J. Chen, M. Chen, Dynamic obstacle avoidance for manipulators using distance calculation and discrete detection, Robotics and Computer-Integrated Manufacturing 49(Supplement C) (2018) 98-104.

43. P. D. H. Nguyen et al., A fast heuristic Cartesian space motion planning algorithm for many-DoF robotic manipulators in dynamic environments, in IEEE-RAS 16th Int. Conf. Humanoid Robots (Humanoids)(Cancun, Mexico, 2016), pp. 884-891.

44. J. Vannoy, J. Xiao, Real-Time Adaptive Motion Planning (RAMP) of Mobile Manipulators in Dynamic Environments With Unforeseen Changes, IEEE Trans. Robot. 24(5) (2008) 1199-1212.

45. Zhao, J., Wei, Y. (2017), A Novel Algorithm of Human-Like Motion Planning for Robotic Arms, International Journal of Humanoid Robotics 14(01) (2017), 1650023.

46. Wu, Q. C., Wang, X. S., Du, F. P., Analytical inverse kinematic resolution of a re- 
dundant exoskeleton for upper-limb rehabilitation, International Journal of Humanoid Robotics 13(03) (2016) 1550042.

47. C. Lamperti, A. M. Zanchettin, P. Rocco, A redundancy resolution method for an anthropomorphic dual-arm manipulator based on a musculoskeletal criterion, in IEEE/RSJ Int. Conf. Intelligent Robots and Systems (IROS) (Hamburg, Germany, 2015), pp. 1846-1851.

48. S. Nishiguchi et al., Theatrical approach: Designing human-like behaviour in humanoid robots, Robotics and Autonomous Systems 89 (2017) 158-166.

49. M. M. Emamzadeh, N. Sadati, W. A. Gruver, Fuzzy-based interaction prediction approach for hierarchical control of large-scale systems, Fuzzy Sets and Systems 329(Supplement C) (2017) 127-152.

50. Y. Qian, Design and Control of a Personal Assistant Robot, Ph.D. thesis (2013).

51. J. H. Holland, Adaptation in natural and artificial systems: an introductory analysis with applications to biology, control, and artificial intelligence, (MIT press, 1992).

52. B. Xian et al., Task-Space Tracking Control of Robot Manipulators via Quaternion Feedback, IEEE Trans. Robot. Autom. 20(1) (2004) 160-167.

53. L. Sciavicco, B. Siciliano, Modelling and Control of Robot Manipulators, Advanced textbooks in control and signal processing, 2nd edn. (London, Springer, 2005).

54. S. McLeod, J. Xiao, Real-time adaptive non-holonomic motion planning in unforeseen dynamic environments, in IEEE/RSJ Int. Conf. Intelligent Robots and Systems $(I R O S)$ (Daejeon, South Korea, 2016), pp. 4692-4699.

55. D. Berenson, S. Srinivasa, J. Kuffner, Task Space Regions: A framework for poseconstrained manipulation planning, The International Journal of Robotics Research 30(12) (2011) 1435-1460.

56. M. Greiff, A. Robertsson, Optimisation-based motion planning with obstacles and priorities, IFAC-PapersOnLine 50(1) (2017) 11670-11676. 


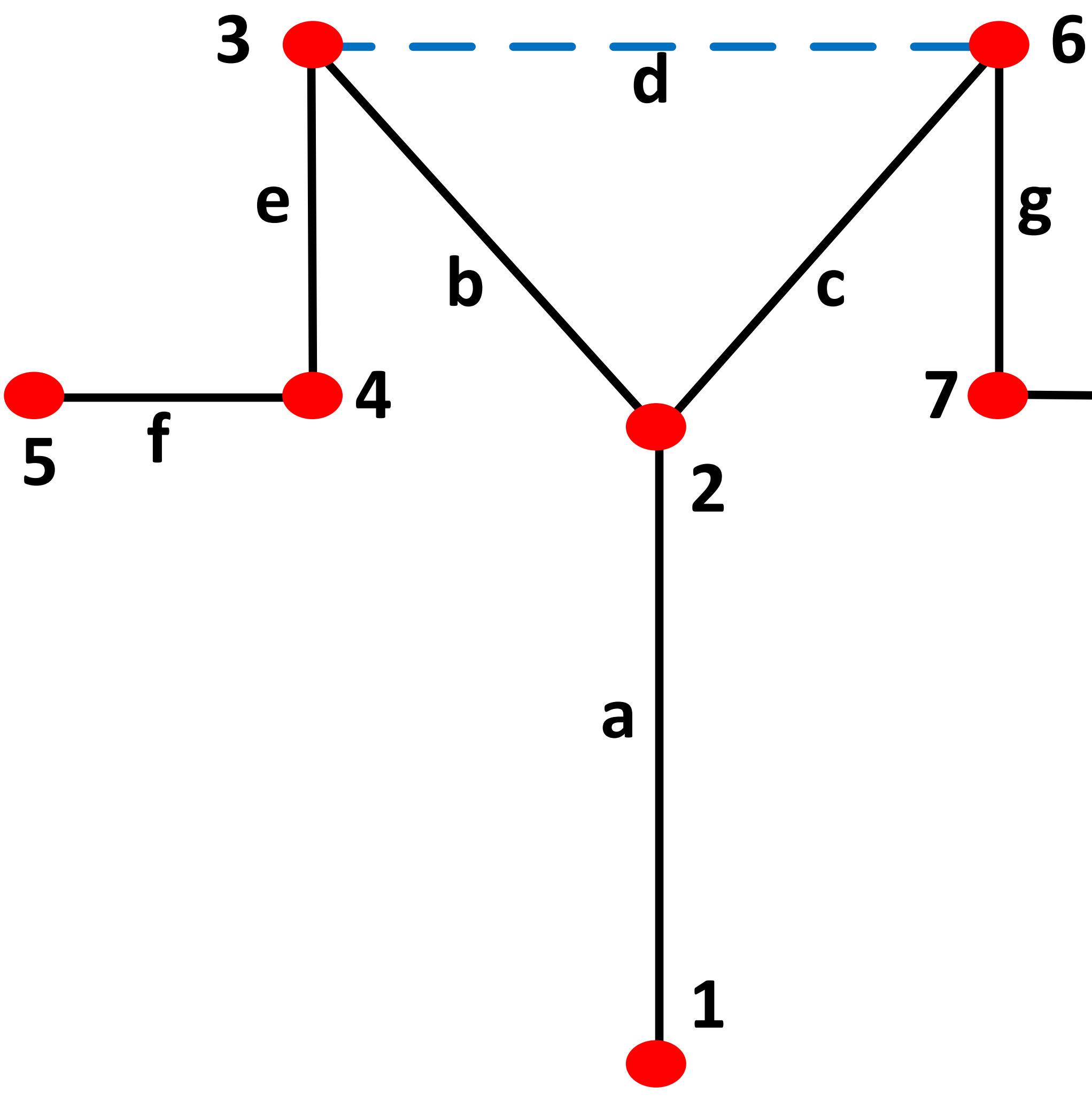

